Article

\title{
All-Ceramic Single Crown Restauration of Zirconia Oral Implants and Its Influence on Fracture Resistance: An Investigation in the Artificial Mouth
}

\section{Ralf-Joachim Kohal ${ }^{1, \dagger}$, Jolanta Bernadette Kilian ${ }^{2, \dagger}$, Susanne Stampf ${ }^{3, \dagger}$ and Benedikt Christopher Spies ${ }^{1, *}$}

1 Department of Prosthetic Dentistry, Center for Dental Medicine, University Hospital Freiburg, Albert-Ludwigs-University, Freiburg 79106, Germany; E-Mail: ralf.kohal@uniklinik-freiburg.de

2 Private Practice, Singen 78224, Germany; E-Mail: jolanta.kilian@uniklinik-freiburg.de

3 Department for Medical Biometry and Medical Informatics, Institute for Medical Biometry and Statistics, Albert-Ludwigs-University, Freiburg 79104, Germany; E-Mail: Susanne.Stampf@usb.ch

$\dagger$ These authors contributed equally to this work.

* Author to whom correspondence should be addressed; E-Mail: benedikt.spies@uniklinik-freiburg.de; Tel.: +49-761-270-47680; Fax: +49-761-270-49250.

Academic Editor: Ihtesham ur Rehman

Received: 23 February 2015 / Accepted: 27 March 2015 / Published: 1 April 2015

\begin{abstract}
The aim of the current investigation was to evaluate the fracture resistance of one-piece zirconia oral implants with and without all-ceramic incisor crowns after long-term thermomechanical cycling. A total of 48 implants were evaluated. The groups with crowns (C, 24 samples) and without crowns (N, 24 samples) were subdivided according to the loading protocol, resulting in three groups of 8 samples each: Group " 0 " was not exposed to cyclic loading, whereas groups " 5 " and " 10 " were loaded with 5 and 10 million chewing cycles, respectively. This resulted in 6 different groups: C0/N0, C5/N5 and C10/N10. Subsequently, all 48 implants were statically loaded to fracture and bending moments were calculated. All implants survived the artificial aging. For the static loading the following average bending moments were calculated: C0: $326 \mathrm{Ncm}$; C5: $339 \mathrm{Ncm}$; C10: $369 \mathrm{Ncm}$; N0: $339 \mathrm{Ncm}$; N5: $398 \mathrm{Ncm}$ and N10: $355 \mathrm{Ncm}$. To a certain extent, thermomechanical cycling resulted in an increase of fracture resistance which did not prove to be statistically significant. Regarding its fracture resistance, the evaluated ceramic implant system made of
\end{abstract}


Y-TZP seems to be able to resist physiological chewing forces long-term. Restauration with all-ceramic single crowns showed no negative influence on fracture resistance.

Keywords: zirconia implants; Y-TZP; fracture load; bending moment; artificial ageing

\section{Introduction}

The clinical long-term results reported for oral implants made of titanium and its biomedical alloys [1,2] have made titanium the "gold standard" material for their fabrication. Besides its favorable physical and mechanical properties [3], titanium shows a high biocompatibility and a low potential of corrosion [4]. Nevertheless, there have been some concerns that titanium might evoke an unwelcome host reaction but the significance of titanium as a cause of allergic reactions in patients with dental implants remains unproven [5-9]. Notwithstanding, the rising popularity of metal-free reconstructions motivates clinicians to offer an implant made of another material than titanium. Its predominant biomechanical behavior among biomedical ceramics makes zirconium dioxide to be the ceramic of choice for the fabrication of dental implants [10]. It has been described that zirconium dioxide exhibits no cytotoxic, sensitizing, mutagenic or oncogenic effect [11-18]. Primarily, Yttria-stabilized zirconia (Yttria-stabilized tetragonal zirconia polycrystal $=\mathrm{Y}$-TZP) seems to be the favorable core material for the manufacturing of dental implants. This material is characterized by a dense, monocrystalline homogeneity, possesses a low thermal conductivity, a low corrosion potential and a good radiopacity [19]. Y-TZP exhibits high flexural strength values $(900-1200 \mathrm{MPa})$ and fracture toughness $\left(9-10 \mathrm{MPa}^{0.5}\right.$ ) owing to a phase transformation toughening mechanism [20]. Nevertheless, aqueous induced dissolution and phase changes of zirconia ceramics resulting in mechanical strength degradation (also known as low-temperature degradation; LTD) are a major concern regarding the clinical long-term performance of Y-TZP [21]. The stability and durability of prepared and unprepared one-piece dental implants made of Y-TZP after up to 20 years of masticatory simulation seems to range within the limits of clinical acceptance [22-24]. Regrettably, none of the mentioned investigations evaluated the influence of a restoration on the fracture-resistance of zirconia oral implants, which resembles clinical reality. Currently available literature for zirconia oral implants with single crown restorations is limited to experimental implant systems over a simulated time period of 5 years with an exerted load of $45-50 \mathrm{~N}[25,26]$. Therefore, the purpose of the current investigation was to evaluate the thermomechanical stability of a market-ready one-piece zirconia implant system with and without all-ceramic single crown reconstructions before and after artificial loading conditions with an exerted load of $98 \mathrm{~N}$ in an aqueous environment over a simulated time period of 20 and 40 years, respectively. Non-loaded samples served as control.

\section{Materials and Methods}

\subsection{Grouping and Sample Preparation}

A total of 48 "zit-z" zirconia dental implants $(4.0 \mathrm{~mm}$ diameter, $13 \mathrm{~mm}$ intraosseous length, $2.5 \mathrm{~mm}$ transgingival height, $4 \mathrm{~mm}$ abutment height; Ziterion $\mathrm{GmbH}$, Uffenheim, Germany) were used for the experiment (Figure 1). Material properties, as indicated by the manufacturing company and according 
to ISO 13356, are shown in Table 1. The implants were equally divided into two groups (Table 2). Group C consisted of 24 implants with all-ceramic (IPS e.max ${ }^{\circledR}$ CAD; Ivoclar Vivadent, Schaan, Liechtenstein) incisor single crown restorations. The 24 implants of group N were used "as delivered". Both groups were further divided into three subgroups: subgroup " 0 " - eight implants that were not subjected to artificial loading in the chewing machine; subgroup " 5 " - eight implants that were subjected to 5 million loading cycles in an aqueous environment in the artificial mouth and subgroup "10" - eight implants that were subjected to 10 million loading cycles in an aqueous environment in the artificial mouth. In the end 6 different groups for evaluation resulted: C0, C5, C10, N0, N5 and N10.
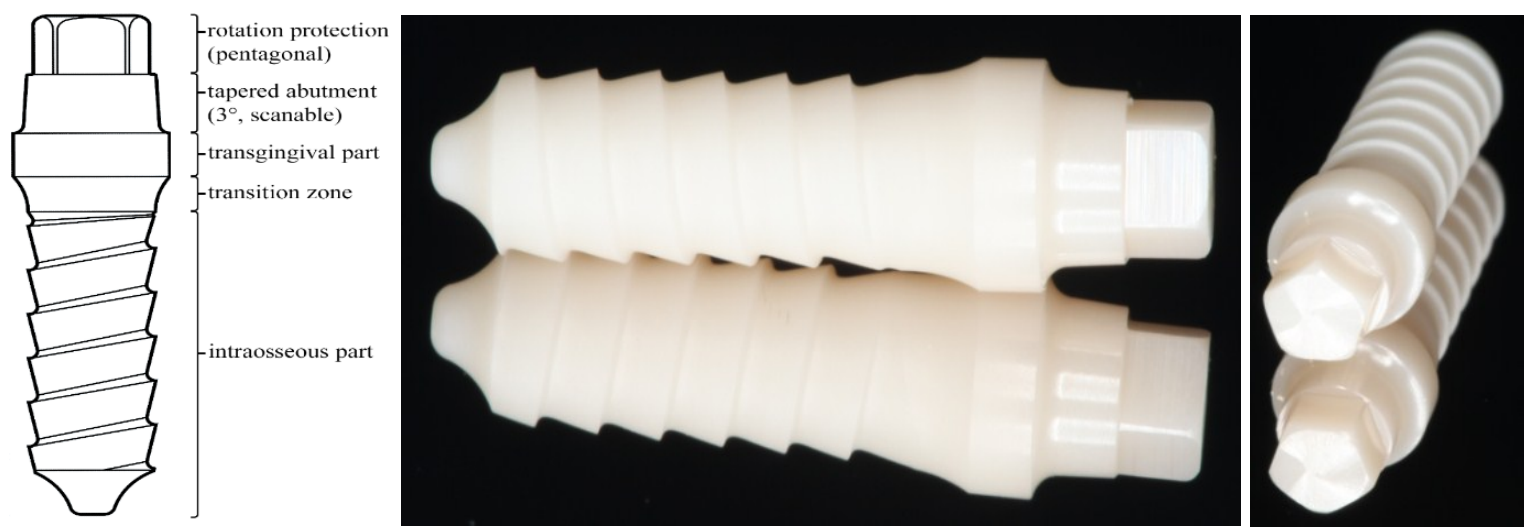

Figure 1. The evaluated implant system (zit-z, Ziterion GmbH, Uffenheim, Germany) and its metrics used for the experiment: $4.0 \mathrm{~mm}$ diameter, $13 \mathrm{~mm}$ intraosseous length, $2.5 \mathrm{~mm}$ transgingival height, $4 \mathrm{~mm}$ abutment height (pentagonal and tapered part).

Table 1. Material properties according to the manufacturer.

\begin{tabular}{ccc}
\hline Characteristics & Unit & Y-TZP \\
\hline Components & & $\mathrm{ZrO}_{2} / \mathrm{Y}_{2} \mathrm{O}_{3}$ \\
Composition & $\mathrm{wt} \%$ & $95 / 5$ \\
Density & $\mathrm{g} / \mathrm{cm}^{3}$ & $>6.0$ \\
Grain size & $\mu \mathrm{m}$ & $<0.6$ \\
Bending strength & $\mathrm{MPa}$ & $>1200$ \\
\hline
\end{tabular}

Table 2. Grouping of test and control specimens.

\begin{tabular}{cccccc}
\hline \multicolumn{4}{c}{ 48 Zirconia Implants } \\
\hline \multicolumn{4}{c}{ Group "C" } \\
$\mathrm{n}=24$ & \multicolumn{4}{c}{ Group "N" } \\
$\mathrm{n}=24$ \\
Implants with single crown restauration & Implants & without restauration ("as delivered") \\
$\mathrm{C} 0$ & $\mathrm{C} 5$ & $\mathrm{C} 10$ & $\mathrm{~N} 0$ & $\mathrm{~N} 5$ & $\mathrm{~N} 10$ \\
$\mathrm{n}=8$ & $\mathrm{n}=8$ & $\mathrm{n}=8$ & $\mathrm{n}=8$ & $\mathrm{n}=8$ & $\mathrm{n}=8$ \\
0 cycles & $5 \times 10^{6}$ cycles & $10 \times 10^{6}$ cycles & 0 cycles & $5 \times 10^{6}$ cycles & $10 \times 10^{6}$ cycles \\
$\downarrow$ & Dynamic & Dynamic & $\downarrow$ & Dynamic & Dynamic \\
& loading & loading & loading & loading \\
& & Static loading test & & \\
\hline
\end{tabular}


All implants were embedded in an autopolymerizing acrylic resin (Technovit ${ }^{\circledR}$ 4000, Heraeus Kulzer, Wehrheim, Germany) into special sample holders at an angle of $45^{\circ}$ to the vertical (Figure 2), replicating the position of upper central incisors [27]. In order to represent a physiological clinical situation after one year [28,29], the implants were embedded 0.5 to $1 \mathrm{~mm}$ above bone level. The resin had a modulus of elasticity of approximately $12 \mathrm{GPa}$ which approximates that of human bone (10-18 GPa) [30].

A wax-up of a central incisor to an implant abutment has been performed (Figure 3a,b). After a scan (inEos, Sirona, Bensheim, Germany) of the bare abutment and the wax-up (Figure 3c/d), 24 monolithic single crowns were finally modified via computer-aided design (Figure $3 \mathrm{e}$ ) and subsequently grinded out of pre-sintered lithium disilicate blanks (IPS e.max ${ }^{\circledR}$ CAD; Figure 3f/g) by computer-aided manufacturing (inLab 3D, Sirona), followed by a final sintering process $\left(30 \mathrm{~min}, 850{ }^{\circ} \mathrm{C}\right.$; Figure $3 \mathrm{~g}-\mathrm{j}$ ). According to manufacturer's instructions for bonding to zirconia, the polished lithium disilicate crowns were adhesively cemented (Multilink Automix, Ivoclar Vivadent) to the abutments of group "C" implants and finally light-cured with a polymerization lamp. Standardized photographs were used to measure the lever arm for each of the 48 samples (Figure 4).
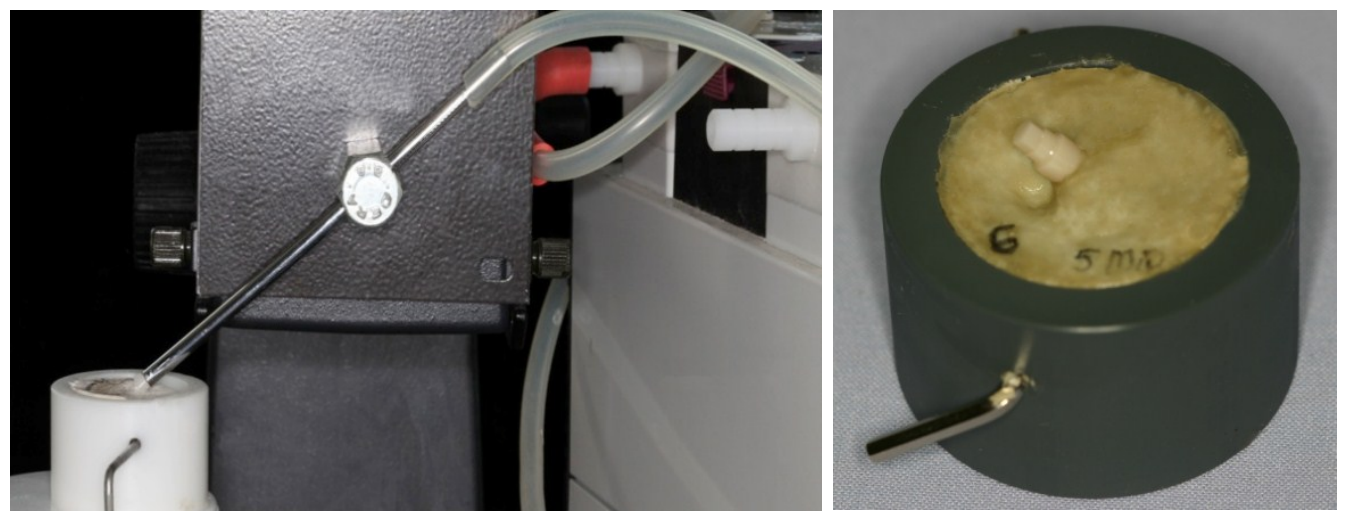

Figure 2. Embedding of implants at an angle of $45^{\circ}$ to the vertical with the help of a vacuum pump to fix the samples in place.
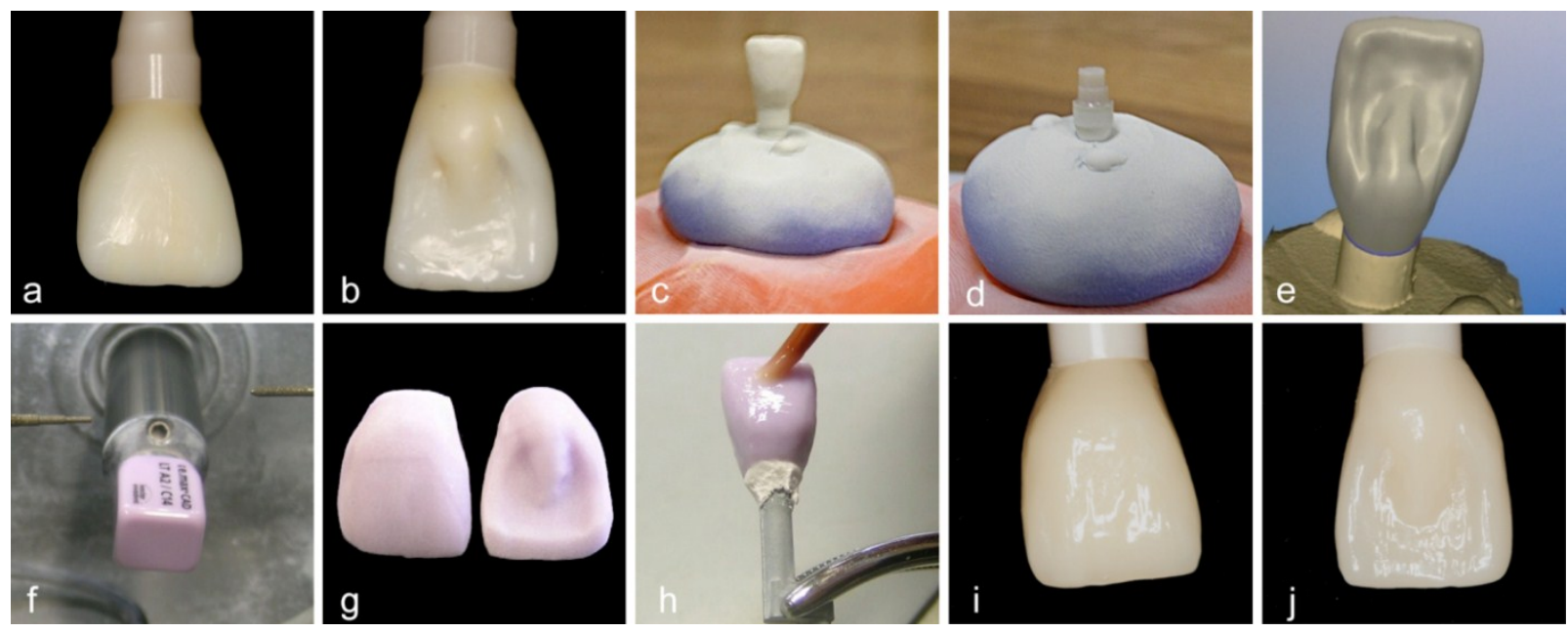

Figure 3. Manufacturing process of the incisor single crowns: Wax-up to implant abutment (a/b); Scan of Wax-up (c) and abutment (d); Computer-aided design (e); Computer-aided manufacturing (f) and restoration before glazing (g); Glazing (h); Final restoration $(\mathbf{i} / \mathbf{j})$. 

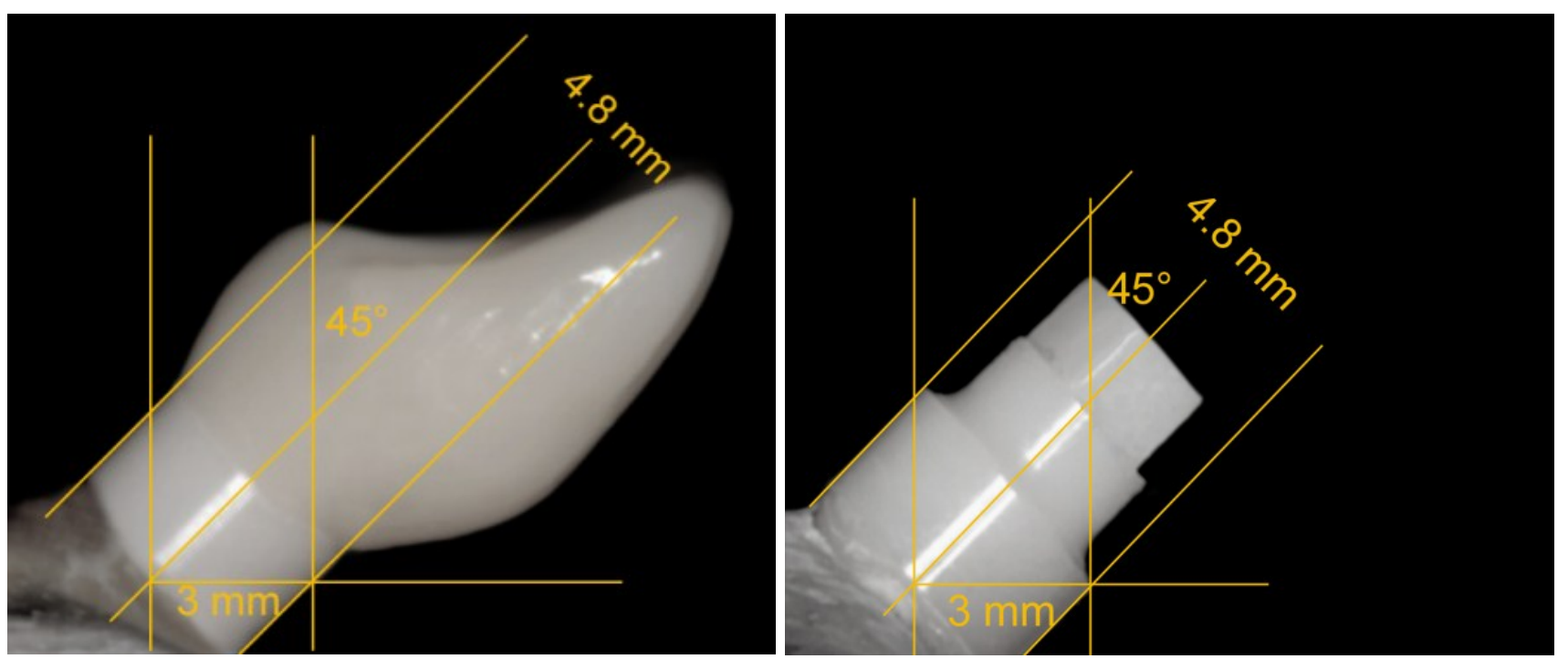

Figure 4. Standardized photographs of the embedded implants with (left; groups C) and without (right; groups N) restoration allowed the measurement of the lever arm (the diameter of the transgingival platform served as reference).

\subsection{Dynamic Loading Test}

Thirty-two of the specimens were thermomechanically aged in a computer-controlled dual axis-chewing simulator in an aqueous environment (Willytec, Munich, Germany; Figure 5) in order to simulate twenty years (5 million cycles; subgroups C5/N5) and almost forty years (10 million cycles; subgroups $\mathrm{C} 10 / \mathrm{N} 10)$ of clinical service, assuming an annual masticatory performance of 240,000-250,000 occlusal contacts [31]. The chewing simulator-environment consisted of eight identical sample chambers filled with water, two stepper motors controlling vertical and horizontal movements of the antagonists (Steatit ${ }^{\circledR}$ ceramic balls, $6 \mathrm{~mm}$ in diameter, Hoechst Ceram Tec, Wunsiedel, Germany) against the implant samples, and a hot and cold water circulation system (Haake, Karlsruhe, Germany). The antagonist ball had a Vickers hardness similar to that of enamel [32]. The applied load in the chewing simulator was $98 \mathrm{~N}(10 \mathrm{~kg})[33,34]$ and the point of load application on the implants was placed on the palatal of the single crowns (groups "C") and the upper edge of the implant abutments (groups "N"), respectively. The load was applied onto the implants by combined vertical (6 mm) and horizontal $(0.5 \mathrm{~mm})$ movements, which—via computerized adaptation-represented an approximation to the physiological masticatory cycle of axial pressure and horizontal shear. The cyclic loading was set at $1.6 \mathrm{~Hz}$. The thermocycling was from $5{ }^{\circ} \mathrm{C}$ to $55^{\circ} \mathrm{C}$ for $60 \mathrm{~s}$ each with an intermediate pause of $12 \mathrm{~s}$, maintained by the thermostatically-controlled liquid circulator (Haake, Karlsruhe, Germany). During the dynamic loading, all samples were examined twice a day. The chewing machine needed approximately 36 and 72 days to accomplish 5 and 10 million cycles, respectively. Fractures of the implants were recorded as a failure. The details of the settings of the chewing simulator machine are listed in Table 3. 


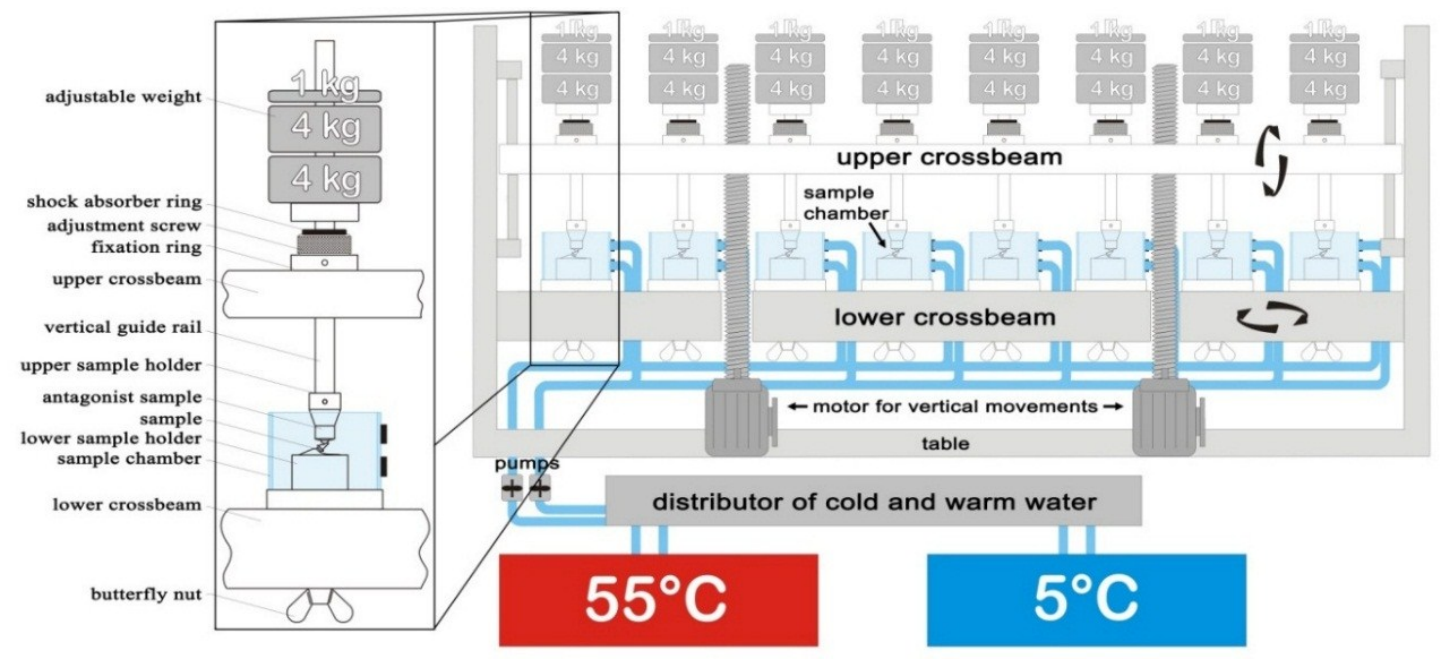

Figure 5. Schematic drawing of the chewing simulator (Willytec, Munich, Germany). The vertical guide rail and the sample holder weigh another $1 \mathrm{~kg}$.

Table 3. Settings of the chewing simulator machine.

\begin{tabular}{cc}
\hline Chewing Cycles & $\mathbf{5 , 0 0 0 , 0 0 0 / 1 0 , 0 0 0 , 0 0 0}$ \\
\hline Cycle frequency & $1.6 \mathrm{~Hz}$ \\
Vertical movement & $6 \mathrm{~mm}$ \\
Horizontal movement & $0.5 \mathrm{~mm}$ \\
Descending speed & $60 \mathrm{~mm} / \mathrm{s}$ \\
Rising speed & $55 \mathrm{~mm} / \mathrm{s}$ \\
Forward speed & $60 \mathrm{~mm} / \mathrm{s}$ \\
Backward speed & $55 \mathrm{~mm} / \mathrm{s}$ \\
Applied weight per sample & $10 \mathrm{~kg}(98 \mathrm{~N})$ \\
Hot dwell time & $60 \mathrm{~s}$ \\
Hot bath temperature & $55^{\circ} \mathrm{C}$ \\
Cold dwell time & $60 \mathrm{~s}$ \\
Cold bath temperature & $5^{\circ} \mathrm{C}$ \\
Intermediate pause & $12 \mathrm{~s}$ \\
\hline
\end{tabular}

\subsection{Static Loading Test}

All samples that survived the exposure to the chewing simulator were statically loaded using a universal-testing machine (Zwick, Z010/TN2S, Ulm, Germany) until fracture occurred. All samples were loaded at the same contact point used for the dynamic loading. A vertical compressive load was applied on the palatal side of the angulated implants under a crosshead speed of $10 \mathrm{~mm} / \mathrm{min}$. The loads required for fracturing the samples were recorded using the X-Y writer of the Zwick testXpert ${ }^{\circledR}$ V 7.1 software, with failure recorded at the first sharp drop-down of the graphical curve. The recorded data were automatically analyzed and a graph was drawn for each sample.

\subsection{Statistical Analysis}

A linear model was fitted to evaluate the effects of restoration type $(\mathrm{C}=$ single crown, $\mathrm{N}=$ no restoration) and loading protocol $(0,5$ and 10 million chewing cycles $)$ on the response variables 
(fracture load and bending moment). This analysis was performed separately for each response variable. Least-square means with $95 \%$ confidence intervals were derived from these models. Thus, the continuous response variables were modelled as a function of restoration type, loading protocol and their interaction. Overall tests for main and interaction were done as well as pairwise comparisons between the levels of the explanatory variables and interaction. Therefore, the method of Tukey-Kramer was applied to adjust for multiple testing. The level of significance was set to 0.05 . Differences of least-square means with corresponding $95 \%$ confidence intervals were plotted. Furthermore, boxplots were used for the graphical presentation of the data. All computations were performed with the statistical software SAS (SAS system v9.1.2; SAS Institute Inc., Cary, NC, USA) using PROC MIXED.

\section{Results}

\subsection{Dynamic Loading Test}

All 24 artificially loaded implants survived the dynamic loading test resulting in $100 \%$ survival. However, five single crowns of group $\mathrm{C} 10$ had to be re-cemented during the dynamic loading test. One crown of group C10 fractured after 4.5 million chewing cycles and had to be replaced. Therefore, the survival rate for the restoration was $100 \%$ for group $\mathrm{C} 5$ and $87.5 \%$ for group $\mathrm{C} 10$. All single crowns of group $\mathrm{C} 5 / \mathrm{C} 10$ showed a distinct wear of the palatal contact point. Regarding the loading protocol as well as the restoration type, the lever arms showed no major alterations $(2.9-3.0 \mathrm{~mm})$. Therefore, the exerted bending moment on the samples during the dynamic loading test was roughly equivalent for all samples $(28.4-29.4 \mathrm{Ncm})$.

\subsection{Static Loading Test}

The pattern of fracture for the implants is shown in Figure 6: Independent of the presence of a restoration, all implants fractured slightly below the embedding margin between the first and the second thread. The mean values of the fracture load and bending moment are listed in Table 4. Furthermore, the calculated values of the fracture load and the bending moment were illustrated in the form of box plots (Figure 7).

Table 4. Means of measurements (fracture load, bending moment) for the different test groups ( $\mathrm{n}=$ number of specimens).

\begin{tabular}{|c|c|c|c|c|c|}
\hline \multirow[t]{2}{*}{ Subgroup } & \multirow[t]{2}{*}{$n$} & \multicolumn{2}{|c|}{$\begin{array}{l}\text { Fracture load }[\mathrm{N}] \\
\text { F }\end{array}$} & \multicolumn{2}{|c|}{$\begin{array}{c}\text { Bending moment }[\mathrm{Ncm}] \\
M\end{array}$} \\
\hline & & mean & $S D$ & mean & $S D$ \\
\hline $\mathrm{C} 0$ & 8 & 1095.2 & 183.4 & 325.6 & 53.3 \\
\hline $\mathrm{C} 5$ & 8 & 1131.5 & 189.1 & 339.5 & 56.7 \\
\hline $\mathrm{C} 10$ & 8 & 1230.6 & 109.7 & 369.2 & 32.9 \\
\hline No & 8 & 1130.5 & 245.6 & 339.2 & 73.7 \\
\hline N5 & 8 & 1336.6 & 110.4 & 397.5 & 32.1 \\
\hline N10 & 8 & 1184.4 & 147.0 & 355.3 & 44.1 \\
\hline
\end{tabular}




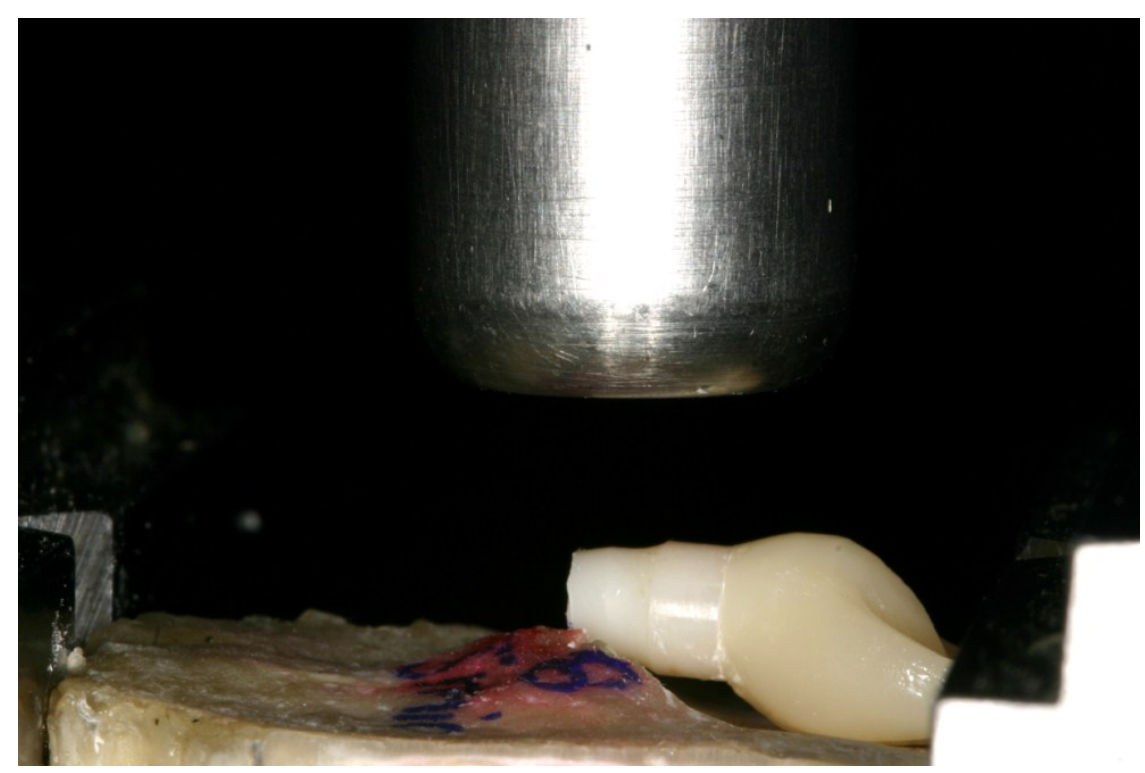

Figure 6. Fracture of all samples occurred between the first and the second thread slightly below the embedding margin.
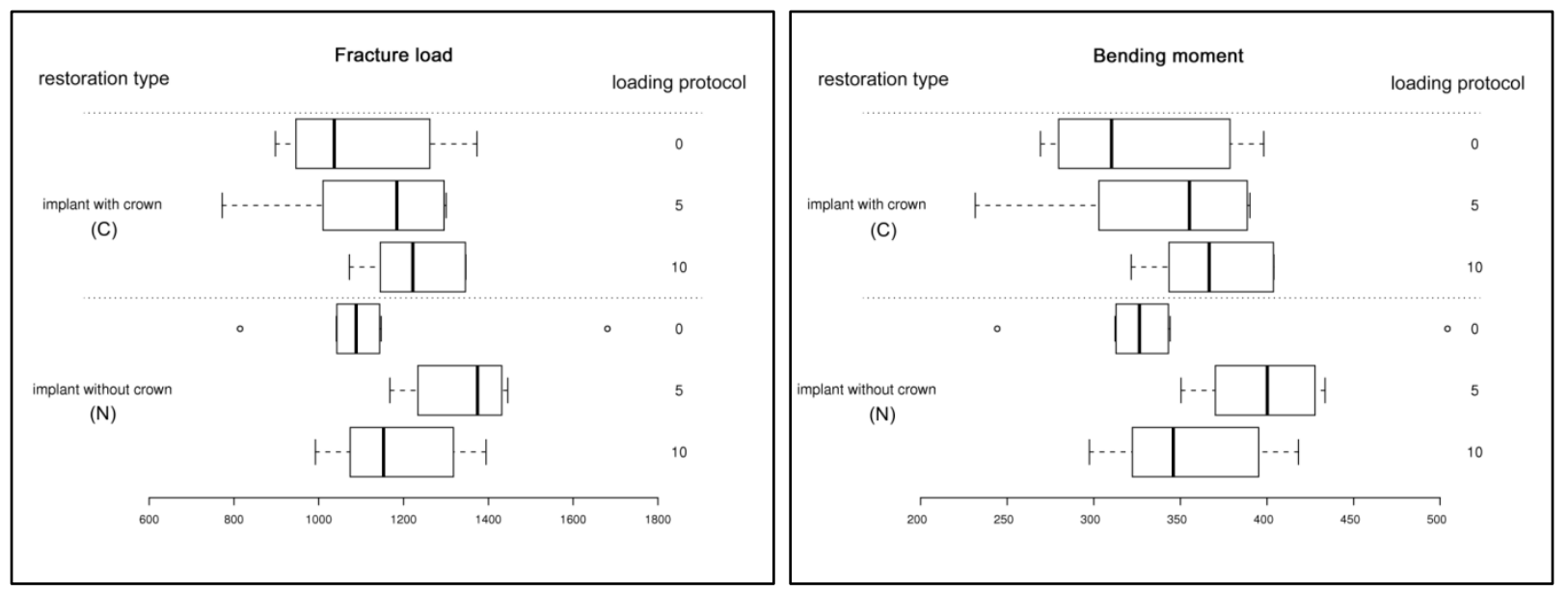

Figure 7. Box plot diagrams of the fracture load in [N] (left) and the bending moment in [Ncm] (right) sorted by restoration type (C: implant with crown; $\mathrm{N}$ : implant without crown) and loading protocol ( 0,5 and 10 million chewing cycles).

\subsection{Statistical Analysis}

For the bending moment and the fracture load, the overall test revealed no statistically significant differences regarding the restoration type ( $\mathrm{C}$ : with single crown restoration; $\mathrm{N}$ : no restoration), loading protocol (0, 5 and 10 million chewing cycles) and their interaction. Furthermore, pairwise comparisons between the levels of the explanatory variables and interaction showed no significance after adjustment (Tukey-Kramer; Table 5). 
Table 5. Pairwise comparison (adjustment: Tukey-Kramer) of subgroups regarding their response variables (bending moment and fracture load).

\begin{tabular}{|c|c|c|c|c|}
\hline \multirow{2}{*}{ Comparison } & \multicolumn{2}{|c|}{ Bending moment } & \multicolumn{2}{|c|}{ Fracture load } \\
\hline & adj. $p$-value & Significance & adj. $p$-value & Significance \\
\hline $\mathrm{C} 0: \mathrm{C} 5$ & 0.994 & not significant & 0.9981 & not significant \\
\hline $\mathrm{C} 0: \mathrm{C} 10$ & 0.5322 & not significant & 0.6137 & not significant \\
\hline C0 : N0 & 0.9945 & not significant & 0.9983 & not significant \\
\hline $\mathrm{C} 0: \mathrm{N} 5$ & 0.0728 & not significant & 0.0732 & not significant \\
\hline $\mathrm{C} 0: \mathrm{N} 10$ & 0.8497 & not significant & 0.9001 & not significant \\
\hline $\mathrm{C} 5: \mathrm{C} 10$ & 0.8493 & not significant & 0.8537 & not significant \\
\hline C5 : N0 & 1.0 & not significant & 1.0 & not significant \\
\hline C5 : N5 & 0.2239 & not significant & 0.1802 & not significant \\
\hline C5 : N10 & 0.9886 & not significant & 0.989 & not significant \\
\hline C10 : N0 & 0.8439 & not significant & 0.8485 & not significant \\
\hline $\mathrm{C} 10: \mathrm{N} 5$ & 0.8727 & not significant & 0.8146 & not significant \\
\hline $\mathrm{C} 10: \mathrm{N} 10$ & 0.9939 & not significant & 0.9942 & not significant \\
\hline N0 : N5 & 0.2192 & not significant & 0.1761 & not significant \\
\hline N0 : N10 & 0.9876 & not significant & 0.9881 & not significant \\
\hline N5 : N10 & 0.5658 & not significant & 0.4894 & not significant \\
\hline
\end{tabular}

\section{Discussion and Conclusions}

The mechanical properties concerning biomedical zirconia offered by the manufacturers and for instance required to satisfy ISO 13365 are solely based on mechanical strength values and aging kinetics measured on bending bars or discs, which is not simulating clinical reality and not considering any subsequent manufacturing procedures or surface modifications. However, it could be shown that such procedures are liable to compromise the long-term mechanical properties of zirconia or increase its sensitivity to ageing $[35,36]$. Therefore, long-term thermomechanical cycling in an aqueous environment of the market-ready product should be performed in advance to validate the functionality and safety of zirconia implants prior to their clinical use.

To simulate the above mentioned conditions to the extent possible, the experimental setup of the current investigation differed from ISO 14801, which does not include horizontal components of a dynamic loading or any environmental factors. Masticatory simulation trials should imitate the occlusal loading, create forces comparable to those which develop during horizontal and vertical components of masticatory motion and re-create environmental factors such as temperature and moisture fluctuations as found in the oral cavity (Krejci et al. 1994). ISO 14801 dictates the simulation of a $3 \mathrm{~mm}$ bone recession. According to clinical findings after 1 year [28], the implants of the current testing were embedded simulating $0.5-1 \mathrm{~mm}$ of bone recession. Therefore, the calculated pure fracture load values are not comparable to other investigations adapting ISO 14801. Nevertheless, the calculation of the bending moment includes the calculated lever arm in addition to the fracture load and is, therefore, more crucial and the significant value when comparing different investigations, respectively. The load applied to all implants during masticatory simulation was $98 \mathrm{~N}$, and the direction of force between the implant and antagonist was set at $45^{\circ}$ to the vertical. This force $(98 \mathrm{~N})$ was chosen to simulate the physiological loading of maxillary teeth, following a clinical investigation by Fontijn-Tekamp and coworkers who 
found normal chewing forces of 60 to $75 \mathrm{~N}$ in the anterior dentition, and 110 to $125 \mathrm{~N}$ in the posterior dentition [33].

In several in vivo evaluations, maximum bending moments of $27 \mathrm{Ncm}$ have been measured at implant supported fixed partial dentures equipped with strain gauge abutments [37-40]. This value is comparable with the exerted bending moment during the dynamic loading test $(28.4-29.4 \mathrm{Ncm})$. The mean bending moment values at the timepoint of fracture for all groups (326-398 Ncm) exceeded this value to the factor 10 or more. According to the mentioned investigations, the results of the current investigation support the use of the evaluated systems in clinical applications.

Explanation attempts for a transient increase in fracture resistance of Y-TZP after dynamic loading remain theoretical. Nevertheless, all measured deviations of fracture resistance in the current investigation proved to be not statistically significant. It might be a matter of time until the samples of the present investigation, which showed an increase in fracture resistance, reveal decreased fracture resistance due to a proceeding $t \rightarrow m$ phase transformation. There are several in vitro investigations available in the literature focusing on the fracture resistance of zirconia oral implants with and without different modifications (e.g., preparation or restoration) after different loading protocols (e.g., 1.2 to 10 million chewing cycles, with or without thermocycling, 30-98 $\mathrm{N}$ exerted load) presenting increased as well as decreased fracture resistance values after the dynamic loading test [23-25,41,42]. Therefore, the process of zirconia's aging kinetics seems to be not known exactly. Further research and more profound methodology (e.g., Raman spectroscopy and/or X-ray diffraction) after different loading protocols seem to be necessary.

Five restorations (all $\mathrm{C} 10$ ) showed de-cementations during the dynamic loading test after 5 million chewing cycles and had to be re-cemented once. None of the restorations of group $\mathrm{C} 5 / \mathrm{C} 10$ had to be re-cemented before 5 million cycles. Since cement residues were solely on the restoration and not on the abutment, the zirconia/cement interface might be the weak point long-term. The "Metal/Zirconia Primer" was used for cementation according to manufacturer's instructions. Therefore, further long-term investigations exceeding 20 years of masticatory simulation might be necessary to evaluate the optimal material properties and bonding procedures to achieve a more reliable connection between zirconia oral implants and their restorations. Nevertheless, re-cementations could be performed without complications and happened to a timepoint that is in the range of clinical acceptance.

The single crown restorations of the current investigation were grinded out of pre-sintered IPS e.max CAD blank (Ivoclar Vivadent), representing a lithium disilicate ceramic. One of eight crowns of group C10 fractured during the dynamic loading test, resulting in $87.5 \%$ survival for group C10. Regrettably, it might be a coincidence that this sample failed in group C10 and not in group C5 and, therefore, the presented survival rate might be misleading. Due to the failure before 5 million cycles the mentioned sample could have equally been assigned to group C5. Since the sample assignment to the different subgroups was random and before any testing procedure, this interrogation cannot be clarified afterwards. Furthermore, the ball bearing of the affected sample chamber had to be replaced due to deterioration induced malfunction shortly after the fracture occurred. Therefore, it cannot be ruled out that the failure was a result of temporarily uncontrolled forces.

Regarding its fracture resistance, the evaluated ceramic implant system made of Y-TZP seems to be able to resist physiological chewing forces long-term. Restauration with all-ceramic single crowns and different loading protocols in an aqueous environment showed no negative influence on fracture resistance. 


\section{Acknowledgments}

All implants were provided by the manufacturing company (Ziterion $\mathrm{GmbH}$, Uffenheim, Germany). We would like to thank Ivoclar Vivadent for the support in manufacturing the single crowns. Furthermore, the authors would like to thank Dr. Carmen Sauter (Dortmund, Germany) for providing Figure 5.

\section{Author Contributions}

Ralf-Joachim Kohal conceived and designed the experiments; Jolanta Bernadette Kilian performed the experiments; Ralf-Joachim Kohal, Jolanta Bernadette Kilian and Benedikt Christopher Spies analyzed the data; Benedikt Christopher Spies wrote the paper.

\section{Conflicts of Interest}

The authors declare no conflict of interest.

\section{References}

1. Jung, R.E.; Zembic, A.; Pjetursson, B.E.; Zwahlen, M.; Thoma, D.S. Systematic review of the survival rate and the incidence of biological, technical, and aesthetic complications of single crowns on implants reported in longitudinal studies with a mean follow-up of 5 years. Clin. Oral Implants Res. 2012, 23 (Suppl. 6), 2-21.

2. Pjetursson, B.E.; Thoma, D.; Jung, R.; Zwahlen, M.; Zembic, A. A systematic review of the survival and complication rates of implant-supported fixed dental prostheses (fdps) after a mean observation period of at least 5 years. Clin. Oral Implants Res. 2012, 23 (Suppl. 6), 22-38.

3. Niinomi, M. Mechanical properties of biomedical titanium alloy. Mater. Sci. Eng. A 1998, 243, 231-236.

4. Parr, G.R.; Gardner, L.K.; Toth, R.W. Titanium: The mystery metal of implant dentistry. Dental materials aspects. J. Prosthet. Dent. 1985, 54, 410-414.

5. Chaturvedi, T.P. An overview of the corrosion aspect of dental implants (titanium and its alloys). Indian J. Dent. Res. 2009, 20, 91-98.

6. Flatebø, R.S.; Johannessen, A.C.; Grønningsaeter, A.G.; Bøe, O.E.; Gjerdet, N.R.; Grung, B.; Leknes, K.N. Host response to titanium dental implant placement evaluated in a human oral model. J. Periodontol. 2006, 77, 1201-1210.

7. Javed, F.; Al-Hezaimi, K.; Almas, K.; Romanos, G.E. Is titanium sensitivity associated with allergic reactions in patients with dental implants? A systematic review. Clin. Implant Dent. Relat. Res. 2013, 15, 47-52.

8. Sicilia, A.; Cuesta, S.; Coma, G.; Arregui, I.; Guisasola, C.; Ruiz, E.; Maestro, A. Titanium allergy in dental implant patients: A clinical study on 1500 consecutive patients. Clin. Oral Implants Res. 2008, 19, 823-835.

9. Siddiqi, A.; Payne, A.G.; De Silva, R.K.; Duncan, W.J. Titanium allergy: Could it affect dental implant integration? Clin. Or. Implant. Res. 2011, 22, 673-680. 
10. Li, J.; Hastings, G. Oxide bioceramics: Inert ceramic materials in medicine and dentistry. In Handbook of Biomaterial Properties, 1st ed.; Black, J., Hastings, G., Eds.; Springer: London, UK, 1998; pp. 340-354.

11. Kim, D.J.; Lee, M.H.; Lee, D.Y.; Han, J.S. Mechanical properties, phase stability, and biocompatibility of (y, nb)-tzp/al(2)o(3) composite abutments for dental implant. J. Biomed. Mater. Res. 2000, 53, 438-443.

12. Aldini, N.N.; Fini, M.; Giavaresi, G.; Martini, L.; Dubini, B.; Ponzi Bossi, M.G.; Rustichelli, F.; Krajewski, A.; Ravaglioli, A.; Mazzocchi, M.; et al. Osteointegration of bioactive glass-coated and uncoated zirconia in osteopenic bone: An in vivo experimental study. J. Biomed. Mater. Res. 2004, 68A, 264-272.

13. Covacci, V.; Bruzzese, N.; Maccauro, G.; Andreassi, C.; Ricci, G.A.; Piconi, C.; Marmo, E.; Burger, W.; Cittadini, A. In vitro evaluation of the mutagenic and carcinogenic power of high purity zirconia ceramic. Biomaterials 1999, 20, 371-376.

14. Gahlert, M.; Gudehus, T.; Eichhorn, S.; Steinhauser, E.; Kniha, H.; Erhardt, W. Biomechanical and histomorphometric comparison between zirconia implants with varying surface textures and a titanium implant in the maxilla of miniature pigs. Clin. Oral Implants Res. 2007, 18, 662-668.

15. Gahlert, M.; Roehling, S.; Sprecher, C.M.; Kniha, H.; Milz, S.; Bormann, K. In vivo performance of zirconia and titanium implants: A histomorphometric study in mini pig maxillae. Clin. Oral Implants Res. 2012, 23, 281-286.

16. Gahlert, M.; Röhling, S.; Wieland, M.; Sprecher, C.M.; Kniha, H.; Milz, S. Osseointegration of zirconia and titanium dental implants: A histological and histomorphometrical study in the maxilla of pigs. Clin. Oral Implants Res. 2009, 20, 1247-1253.

17. Kohal, R.J.; Wolkewitz, M.; Hinze, M.; Han, J.S.; Bachle, M.; Butz, F. Biomechanical and histological behavior of zirconia implants: An experiment in the rat. Clin. Oral Implants Res. 2009, 20, 333-339.

18. Schliephake, H.; Hefti, T.; Schlottig, F.; Gedet, P.; Staedt, H. Mechanical anchorage and peri-implant bone formation of surface-modified zirconia in minipigs. J. Clin. Periodontol. 2010, 37, 818-828.

19. Manicone, P.F.; Rossi Iommetti, P.; Raffaelli, L. An overview of zirconia ceramics: Basic properties and clinical applications. J. Dent. 2007, 35, 819-826.

20. Christel, P.; Meunier, A.; Heller, M.; Torre, J.P.; Peille, C.N. Mechanical properties and short-term in vivo evaluation of yttrium-oxide-partially-stabilized zirconia. J. Biomed. Mater. Res. 1989, 23, 45-61.

21. Swain, M.V. Impact of oral fluids on dental ceramics: What is the clinical relevance? Dent. Mater. 2014, 30, 33-42.

22. Andreiotelli, M.; Kohal, R.J. Fracture strength of zirconia implants after artificial aging. Clin. Implant Dent. Relat. Res. 2009, 11, 158-166.

23. Kohal, R.J.; Wolkewitz, M.; Tsakona, A. The effects of cyclic loading and preparation on the fracture strength of zirconium-dioxide implants: An in vitro investigation. Clin. Oral Implants Res. 2011, 22, 808-814.

24. Silva, N.R.; Coelho, P.G.; Fernandes, C.A.; Navarro, J.M.; Dias, R.A.; Thompson, V.P. Reliability of one-piece ceramic implant. J. Biomed. Mater. Res. Part B Appl. Biomater. 2009, 88, 419-426. 
25. Kohal, R.J.; Finke, H.C.; Klaus, G. Stability of prototype two-piece zirconia and titanium implants after artificial aging: An in vitro pilot study. Clin. Implant Dent. Rel. Res. 2009, 11, 323-329.

26. Kohal, R.J.; Klaus, G.; Strub, J.R. Zirconia-implant-supported all-ceramic crowns withstand long-term load: A pilot investigation. Clin. Oral Implants Res. 2006, 17, 565-571.

27. Rakosi, T.; Jonas, I.; Graber, T. Orthodontic diagnosis. In Cephalometric Analysis, 1st ed.; Rateitschak, K.H., Ed.; Georg Thieme Stuttgart: New York, NY, USA, 1993; p. 198.

28. Cannizzaro, G.; Torchio, C.; Felice, P.; Leone, M.; Esposito, M. Immediate occlusal versus non-occlusal loading of single zirconia implants. A multicentre pragmatic randomised clinical trial. Eur. J. Or. Implantol. 2010, 3, 111-120.

29. Payer, M.; Arnetzl, V.; Kirmeier, R.; Koller, M.; Arnetzl, G.; Jakse, N. Immediate provisional restoration of single-piece zirconia implants: A prospective case series - results after 24 months of clinical function. Clin. Oral Implants Res. 2013, 24, 569-575.

30. Rho, J.Y.; Ashman, R.B.; Turner, C.H. Young's modulus of trabecular and cortical bone material: Ultrasonic and microtensile measurements. J. Biomech. 1993, 26, 111-119.

31. DeLong, R.; Sakaguchi, R.L.; Douglas, W.H.; Pintado, M.R. The wear of dental amalgam in an artificial mouth: A clinical correlation. Dent. Mater. 1985, 1, 238-242.

32. Wassell, R.W.; McCabe, J.F.; Walls, A.W. A two-body frictional wear test. J. Dent. Res. 1994, 73, $1546-1553$.

33. Fontijn-Tekamp, F.A.; Slagter, A.P.; Van Der Bilt, A.; Van, T.H.M.A.; Witter, D.J.; Kalk, W.; Jansen, J.A. Biting and chewing in overdentures, full dentures, and natural dentitions. J. Dent. Res. 2000, 79, 1519-1524.

34. Schindler, H.J.; Stengel, E.; Spiess, W.E. Feedback control during mastication of solid food textures - a clinical-experimental study. J. Prosthet. Dent. 1998, 80, 330-336.

35. Sanon, C.; Chevalier, J.; Douillard, T.; Cattani-Lorente, M.; Scherrer, S.S.; Gremillard, L. A new testing protocol for zirconia dental implants. Dent. Mater. 2015, 31, 15-25.

36. Piconi, C.; Maccauro, G. Zirconia as a ceramic biomaterial. Biomaterials 1999, 20, 1-25.

37. Morneburg, T.R.; Pröschel, P.A. Measurement of masticatory forces and implant loads: A methodologic clinical study. Int. J. Prosthodont. 2002, 15, 20-27.

38. Duyck, J.; van Oosterwyck, H.; Vander Sloten, J.; de Cooman, M.; Puers, R.; Naert, I. Magnitude and distribution of occlusal forces on oral implants supporting fixed prostheses: An in vivo study. Clin. Oral Implants Res. 2000, 11, 465-475.

39. Morneburg, T.R.; Pröschel, P.A. In vivo forces on implants influenced by occlusal scheme and food consistency. Int. J. Prosthodont. 2003, 16, 481-486.

40. Richter, E.J. In vivo horizontal bending moments on implants. Int. J. Oral Maxillofac. Implants 1998, 13, 232-244.

41. Kohal, R.J.; Wolkewitz, M.; Mueller, C. Alumina-reinforced zirconia implants: Survival rate and fracture strength in a masticatory simulation trial. Clin. Oral Implants Res. 2010, 21, 1345-1352.

42. Spies, B.C.; Sauter, C.; Wolkewitz, M.; Kohal, R.J. Alumina reinforced zirconia implants: Effects of cyclic loading and abutment modification on fracture resistance. Dent. Mater. 2015, 31, 262-272.

(C) 2015 by the authors; licensee MDPI, Basel, Switzerland. This article is an open access article distributed under the terms and conditions of the Creative Commons Attribution license (http://creativecommons.org/licenses/by/4.0/). 